\title{
Supporting Information: A CCSD(T)-based 4-body Potential for Water
}

\author{
Apurba Nandi*, 1, a) Chen Qu*, 2, b) Paul L. Houston*,3, c) Riccardo Conte*, 4, d) Qi \\ Yu*, 5, e) and Joel M. Bowman*1, f) \\ 1) Department of Chemistry and Cherry L. Emerson Center for \\ Scientific Computation, Emory University, Atlanta, Georgia 30322, \\ U.S.A. \\ 2) Department of Chemistry \& Biochemistry, University of Maryland, College Park, \\ Maryland 20742, U.S.A. \\ 3) Department of Chemistry and Chemical Biology, Cornell University, Ithaca, \\ New York 14853, U.S.A. and Department of Chemistry and Biochemistry, \\ Georgia Institute of Technology, Atlanta, Georgia 30332, \\ U.S.A \\ 4) Dipartimento di Chimica, Università Degli Studi di Milano, via Golgi 19, \\ 20133 Milano, Italy \\ 5) Department of Chemistry, Yale University, New Haven, Connecticut 06520, \\ U.S.A.
}

(Dated: 29 September 2021)

\footnotetext{
a)Electronic mail: apurba.nandi@emory.edu

b) Electronic mail: cqu12@umd.edu

c) Electronic mail: plh2@cornell.edu

d) Electronic mail: riccardo.conte1@unimi.it

e) Electronic mail: q.yu@yale.edu

f) Electronic mail: jmbowma@emory.edu
} 
The supporting information contains details of the database of tetramer configurations for the 4-b energies, the choice of $\operatorname{CCSD}(\mathrm{T})$ basis based on a basis-set-superposition analysis, precision analysis of the PIP fit, histograms of OO distances and 4-b energies for the heptamer and decamer water clusters, and a table showing 4-b energies for isomers of the water hexamer from the present $\operatorname{CCSD}(\mathrm{T})-\mathrm{F} 12 \mathrm{a} /$ haTZ calculations, the PES, and previously reported TTM4-F/MB-Pol potentials.

Below are figures of the configurations of the water tetramer described in the main text and Table S1 below.
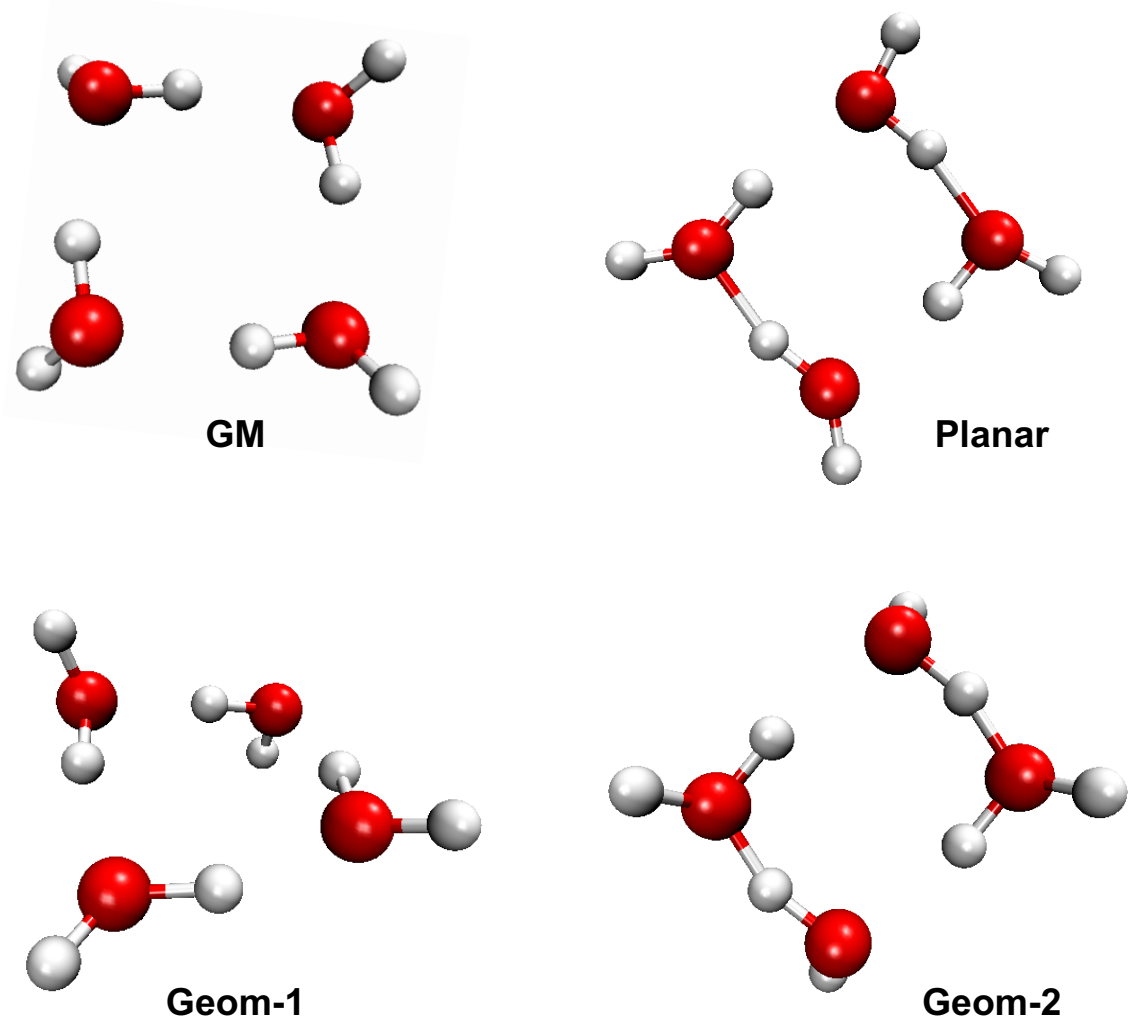

FIG. S1. Water tetramer Geometries.

\section{Database details}

As noted in the main text 468 configurations were recorded from the direct-dynamics calculations. The corresponding tetramer potential energies, relative to the GM, from these calculations are shown in Fig. S2. As seen they span a range from roughly 2000 to 11500 $\mathrm{cm}^{-1}$. This indicates that the tetramer configurations from the AIMD trajectories span a 
large range of distorted geometries.

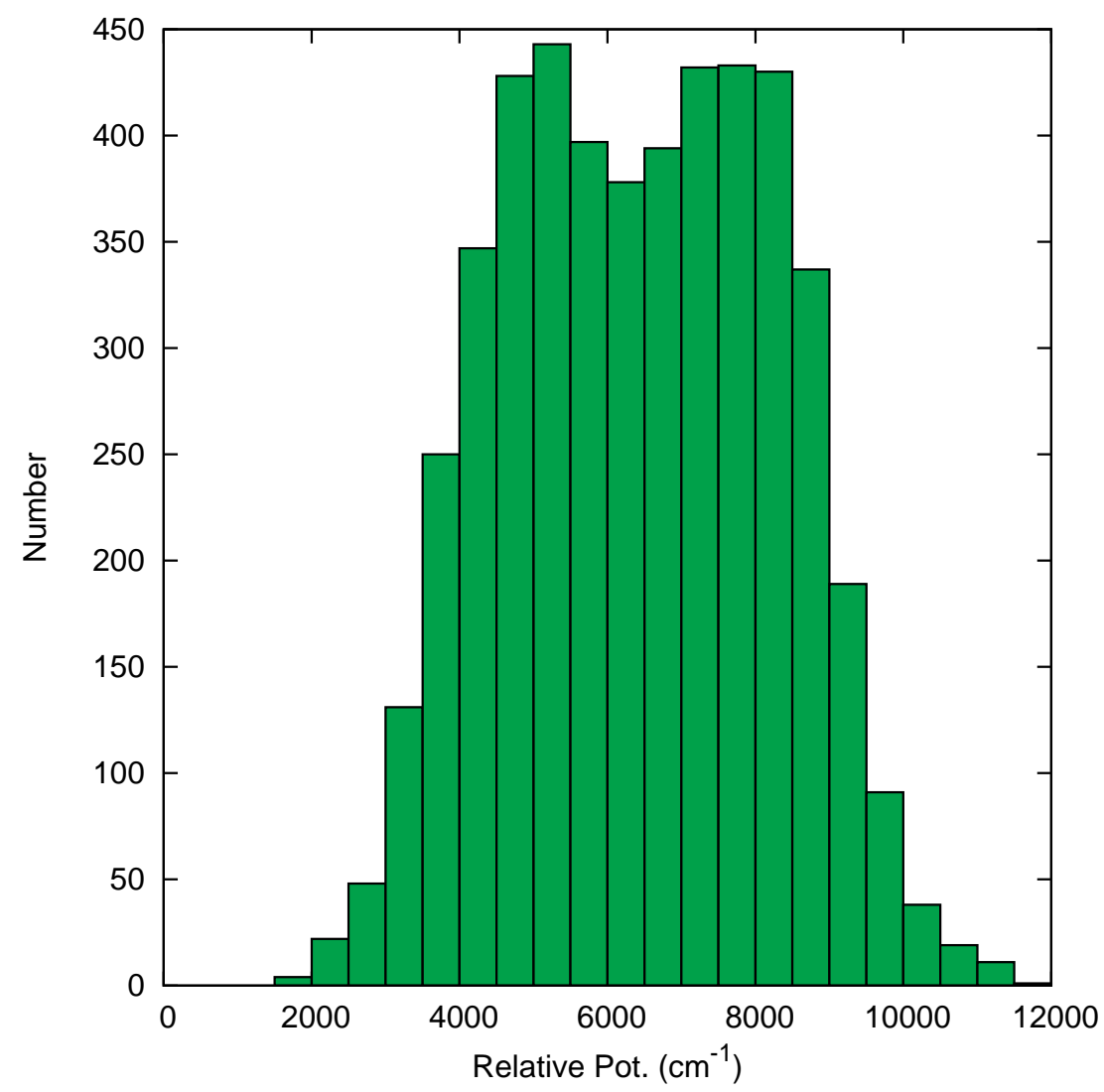

FIG. S2. Tetramer potential energies from direct dynamics trajectories

\section{Choice of Basis for CCSD(T) Calculations}

$\operatorname{CCSD}(\mathrm{T})$ energies were obtained at the configurations generated as just described. The choice of basis was made considering Basis Set Superposition Error (BSSE) and computational efficiency. Since BSSE is typically assessed for interaction energies, that is what we considered for three configurations, the minimum, Geom-1 and Geom-2. Table S1 shows this analysis for three bases. As seen all bases exhibit a relatively small BSSE and the results for the haTZ basis are very close to the much more expensive haQZ basis. So, based on this analysis we chose the haTZ basis for the $\operatorname{CCSD}(\mathrm{T})$ energies at the database configurations. 
TABLE S1. Comparison of BSSE corrected and uncorrected 4-b interaction energies (in $\mathrm{cm}^{-1}$ ) for minimum geometry as well as two more geometries (Geom-1 and Geom-2) at various level of theory.

\begin{tabular}{|c|c|c|c|c|c|c|}
\hline \multirow[b]{2}{*}{ Method } & \multicolumn{2}{|c|}{ Minimum } & \multicolumn{2}{|c|}{ Geom-1 } & \multicolumn{2}{|c|}{ Geom-2 } \\
\hline & corrected & uncorrected & corrected & uncorrected & corrected & uncorrected \\
\hline CCSD(T)-F12a/haDZ & -173.1 & -169.4 & -402.6 & -395.3 & -636.2 & -636.0 \\
\hline CCSD(T)-F12a/haTZ & -174.4 & -174.5 & -405.6 & -404.0 & -647.2 & -646.2 \\
\hline CCSD(T)-F12a/haQZ & -175.9 & -175.3 & -406.8 & -404.8 & -649.4 & -648.7 \\
\hline
\end{tabular}

Fit precision analysis

Fig. S3 shows the correlation plot between $\operatorname{CCSD}(\mathrm{T})$ energies and energies calculated by the PES fit.

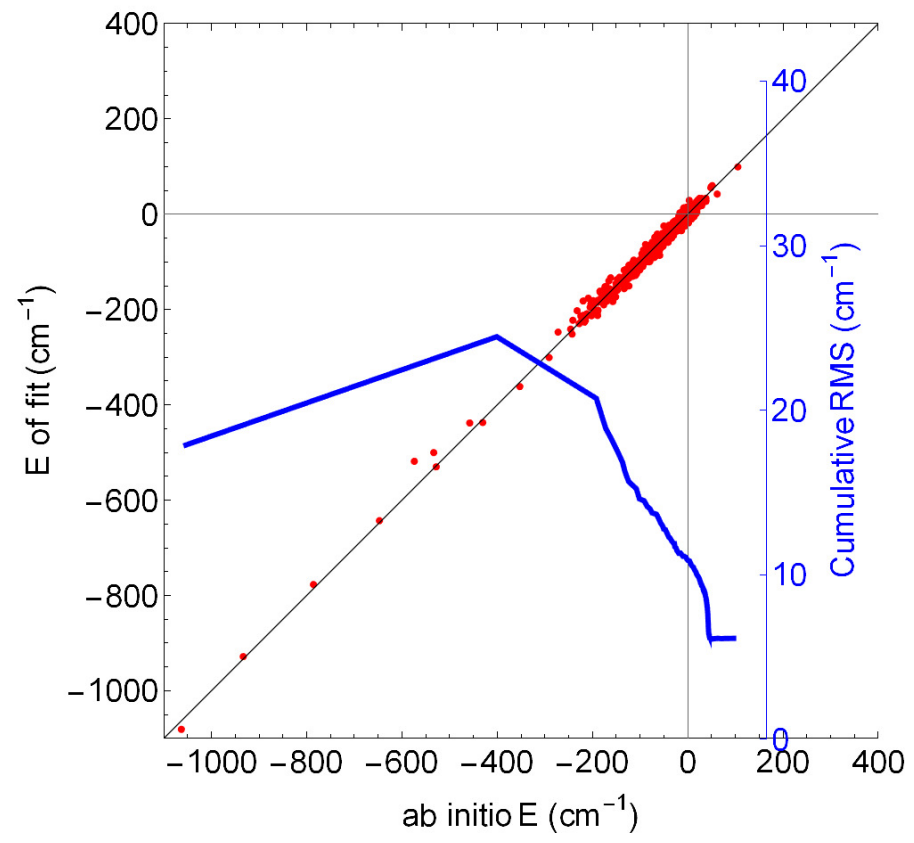

FIG. S3. Correlation plot between ab initio energies and energies calculated by the PES fit. The correlation coefficient is $\mathrm{R}^{2}=0.990$ and the RMS error is $6.2 \mathrm{~cm}^{-1}$. (blue) The cumulative RMS error is in $\mathrm{cm}^{-1}$. 


\section{Histograms of $\mathrm{OO}$ distances and 4-b energies for the heptamer and decamer}

The distribution of $\mathrm{OO}$ distances for the entire dataset is shown as histogram plots in Fig. S4. The distribution of 4-b energies for the water heptamer and decamer are shown in Fig. S5.
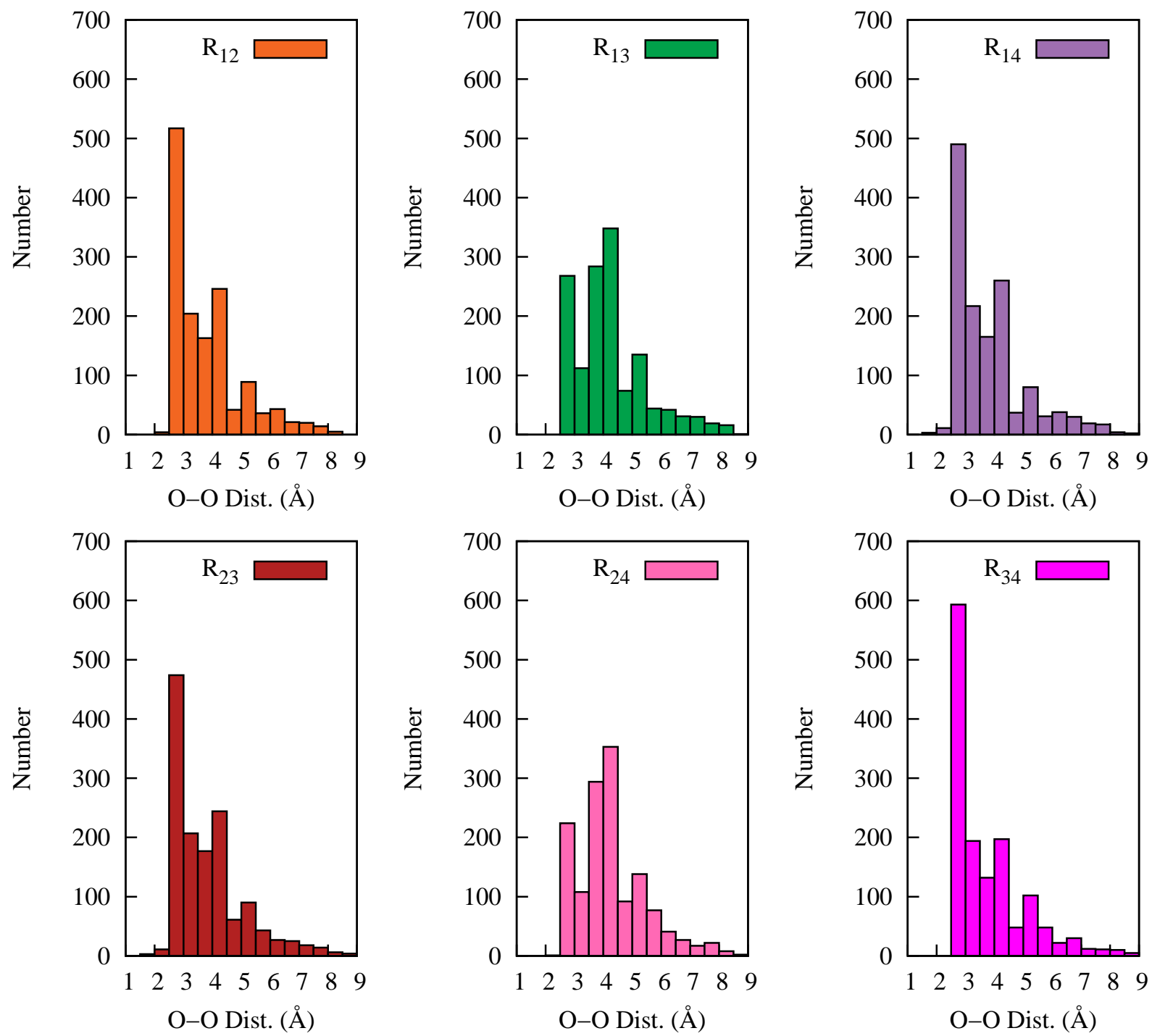

FIG. S4. Distribution of OO distances for the entire dataset. 

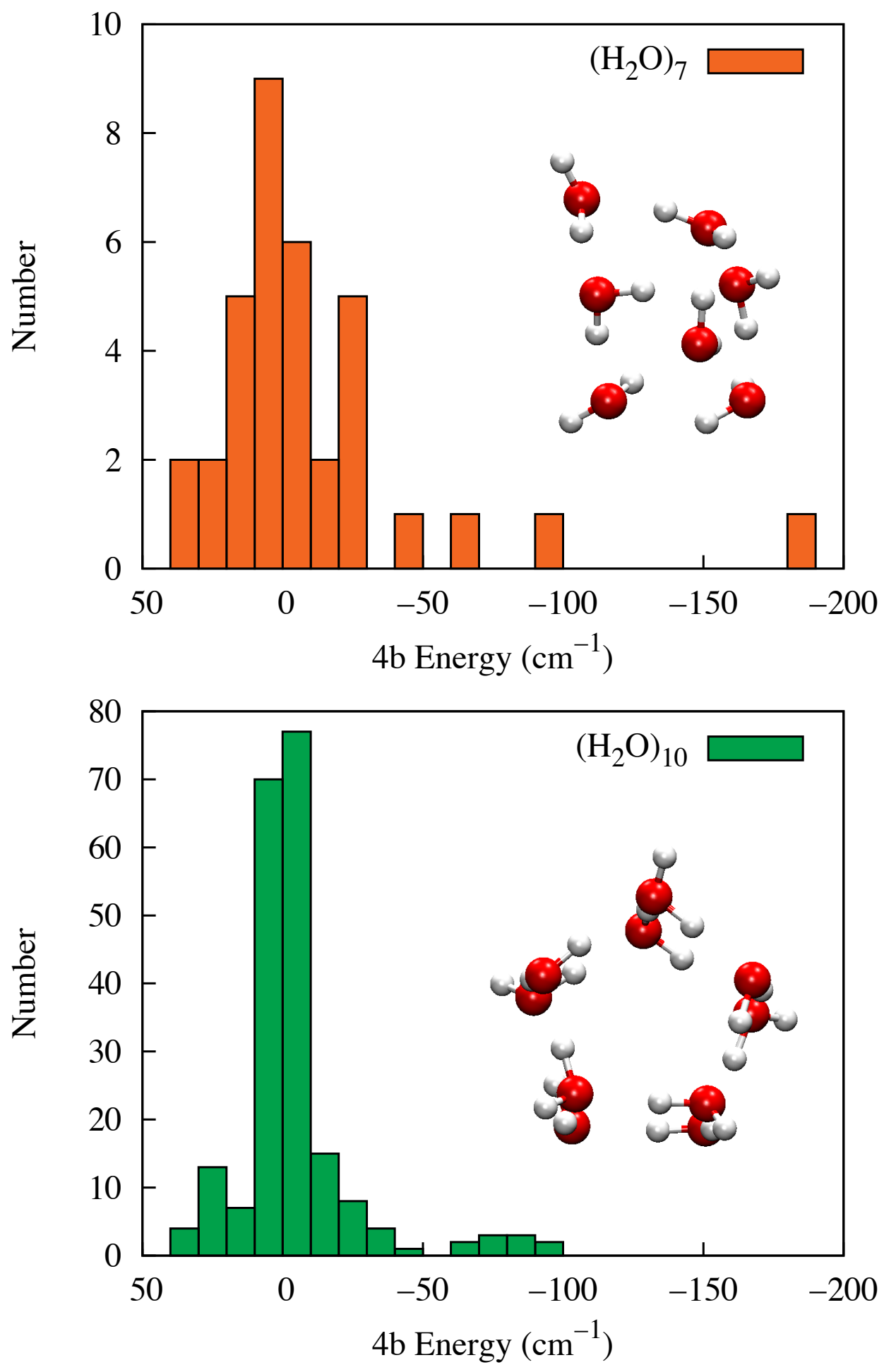

FIG. S5. Histograms of 4-b energies for heptamer and decamer. 


\section{4-b energies of eight isomers of the water hexamer}

TABLE S2. 4-b interaction energies (in $\mathrm{kcal} / \mathrm{mol}$ ) of eight isomers of the water hexamer from indicated sources.

\begin{tabular}{lcccc}
\hline \hline Isomer & PES & $\begin{array}{c}\text { Present CCSD }(\mathrm{T})- \\
\text { F12a/haTZ }\end{array}$ & $\begin{array}{c}\text { CCSD(T) } \\
\text {-F12/VTZ }\end{array}$ & $\begin{array}{c}\text { MB-pol } \\
\text { /TTM4-F }\end{array}$ \\
\hline Prism & -0.66 & -0.62 & -0.66 & -0.52 \\
Cage & -0.61 & -0.54 & -0.53 & -0.47 \\
Book-1 & -1.12 & -1.16 & -1.08 & -0.92 \\
Book-2 & -1.08 & -1.05 & -1.00 & -0.85 \\
Bag & -1.38 & -1.21 & -1.16 & -0.90 \\
Chair & -1.88 & -1.85 & -1.78 & -1.44 \\
Boat-1 & -1.73 & -1.71 & -1.63 & -1.35 \\
Boat-2 & -1.71 & -1.69 & -1.61 & -1.35 \\
\hline \hline
\end{tabular}

${ }^{a}$ From Medders et al J. Chem. Phys. 143, 104102 (2015). 